\title{
'Can you look me in the face?' Short-term SSRI Administration Reverts Avoidant Ocular Face Exploration in Subjects at Risk for Psychopathology
}

\author{
Martina Di Simplicio*, ${ }^{*}, 2$, Sonia Doallo ${ }^{3}$, Giulia Costoloni ${ }^{4}$, Gustavo Rohenkohl ${ }^{5}$, Anna C Nobre ${ }^{5}$ and \\ Catherine J Harmer ${ }^{2}$
}

'MRC Cognition and Brain Sciences Unit, Cambridge, UK; ${ }^{2}$ Department of Psychiatry, University of Oxford, Oxford, UK; ${ }^{3}$ Department of Clinical Psychology and Psychobiology, University of Santiago de Compostela, Santiago de Compostela, Spain; ${ }^{4}$ Neuroscience Department, Psychiatry Section, University of Siena, Siena, Italy; ${ }^{5}$ Department of Experimental Psychology and Oxford Centre for Human Brain Activity, University of Oxford, Oxford, UK

\begin{abstract}
Anxiety and depression are associated with altered ocular exploration of facial stimuli, which could have a role in the misinterpretation of ambiguous emotional stimuli. However, it is unknown whether a similar pattern is seen in individuals at risk for psychopathology and whether this can be modified by pharmacological interventions used in these disorders. In Study I, eye gaze movement during face discrimination was compared in volunteers with high vs low neuroticism scores on the Eysenck Personality Questionnaire. Facial stimuli either displayed a neutral, happy, or fearful expression. In Study 2, volunteers with high neuroticism were randomized in a double-blind design to receive the selective serotonin reuptake inhibitor citalopram $(20 \mathrm{mg})$ or placebo for 7 days. On the last day of treatment, eye gaze movement during face presentation and the recognition of different emotional expressions was assessed. In Study I, highly neurotic volunteers showed reduced eye gaze towards the eyes vs mouth region of the face compared with low neurotic volunteers. In Study 2 , citalopram increased gaze maintenance over the face stimuli compared with placebo and enhanced recognition of positive vs negative facial expressions. Longer ocular exploration of happy faces correlated positively with recognition of positive emotions. Individuals at risk for psychopathology presented an avoidant pattern of ocular exploration of faces. Short-term SSRI administration reversed this bias before any mood or anxiety changes. This treatment effect may improve the capacity to scan social stimuli and contribute to the remediation of clinical symptoms related to interpersonal difficulties.
\end{abstract}

Neuropsychopharmacology (20I4) 39, 3059-3066; doi:I0.I038/npp.20I4.I59; published online I3 August 20I4

\section{INTRODUCTION}

The ability to balance between scanning the environment for signals of potential danger and dismissing negative cues when irrelevant to the current goals is an adaptive function. Anxiety and depression are characterized by aberrant attention to emotional stimuli, involving alterations in ocular exploration of the stimuli. For example, highly anxious individuals can show initial hypervigilance to images appraised as potentially threatening (Armstrong and Olatunji, 2012; Ouimet et al, 2009). This can be followed by avoidance, leading to misattributions in the actual meaning of stimuli and possibly having a crucial role in maintaining symptoms (Armstrong and Olatunji, 2012; Clark and Wells, 1995). In addition, depression has been

*Correspondence: Dr M Di Simplicio, MRC Cognition and Brain Sciences Unit, 15, Chaucer Road, Cambridge CB2 7EF, UK, Tel: + 441223 355294, Fax: + 441223359062

E-mail: martina.disimplicio@mrc-cbu.cam.ac.uk

Received 5 April 20।4; revised 22 June 20।4; accepted 23 June 20।4; accepted article preview online 18 July 2014 associated with a prolonged maintenance of attention over negative pictures (Butler et al, 2008; Caseras et al, 2007; Gotlib et al, 2004) and reduced attention allocation to positive stimuli (Derakshan et al, 2009), consistent with clinical symptoms of overdwelling on negative stimuli (Leyman et al, 2011) and anhedonia.

Avoidance of the processing of social cues such as faces may have knock-on effects on social interactions. The eye region tends to attract attention when people look at faces and is crucial for gender and identity discrimination and for emotion recognition (Nolen-Hoeksema, 2000; Whalen et al, 2004). Biases in eye-region exploration have been mostly investigated in disorders with most evident interpersonal difficulties, such as autism and social phobia (Itier and Batty, 2009). For example, socially anxious individuals showed a hypervigilant-avoidant response to threatening facial expressions, which becomes purely avoidant when put in a socially challenging situation (Garner et al, 2006). Anxiety, interpersonal sensitivity, and dysphoria often cooccur in clinical samples and also in the personality trait of neuroticism, characterized by a predisposition to negative 
affect and increased vulnerability to emotional disorders (Clark and Watson, 1991; Kendler et al, 1993); however, it is poorly understood how this relates to attentional processes towards social stimuli.

Pharmacological treatment of anxiety and depression remains relatively non-specific with selective serotonin reuptake inhibitors (SSRIs) being commonly used in both diagnostic categories (Kent et al, 1998). Antidepressant medication has been shown to modulate the processing of facial stimuli early in treatment in healthy volunteers and depressed patients, promoting a positive pattern of social information processing that is believed to contribute in time to symptom resolution (Pringle et al, 2011). On direct tasks of emotional attention, SSRIs have been shown to decrease vigilance to fearful facial expressions in healthy volunteers (Murphy et al, 2009). However, it is unknown whether SSRIs are also able to modify attention deployment via ocular exploration of facial stimuli early in treatment in samples with negative attentional biases.

This question can be addressed by selecting a population at risk for psychopathology with emotion-processing biases typical of anxiety and depression, such as subjects with high neuroticism traits (Canli, 2008; Ormel et al, 2004). For example, highly neurotic volunteers showed reduced recognition of positive facial expressions of happiness (Chan et al, 2007), and a recent study reported a positive correlation between neuroticism and gaze duration over the eye region of fearful faces (Perlman et al, 2009). Previous studies have also revealed that short-term antidepressant administration is not only able to reverse the negative biases in self-referential processing characterizing vulnerability to depression (Di Simplicio et al, 2012) but can also increase threat-related processing in visual attentional brain areas in the same group (Di Simplicio et al, 2013). However, the relationship between changes in attention towards facial stimuli and changes in negative attentional bias following SSRI treatment in this group has not been investigated despite the potential for important interactions.

The aim of our research was (a) in Study 1, to investigate the presence of attentional biases in ocular exploration of emotional facial expressions (measured by eye-movement tracking) in a population with high and low levels of neuroticism (associated with approximately a threefold difference in risk for depression and anxiety (Kendler et al, 1993)); and (b) in Study 2, to test whether short-term repeated SSRI administration can modulate attentional biases in ocular exploration of emotional facial expressions in the same population at risk for psychopathology, compared with placebo. Given the anxious-depressive phenotype of high neuroticism, we hypothesized that (a) in Study 1, individuals with high neuroticism would present attentional biases in ocular exploration of facial expressions. As previous behavioral data (Browning et al, 2010) and neuroimaging research (Godlewska et al, 2012; Harmer et al, 2006) suggest that short-term repeated SSRI administration can modify initial attention allocation and covert vigilance for negative facial expressions, we also hypothesized that (b) in Study 2, 7 days of SSRI treatment would be able to normalize the biases in ocular exploration of faces associated with neuroticism and (c) this would be correlated with the ability to recognize emotional facial expressions in a separate task.

\section{MATERIALS AND METHODS}

\section{STUDY 1 Ocular Exploration of Emotional Facial Expression: High $v s$ Low Neuroticism}

Volunteers and design. The ethics committee Oxfordshire REC B (Ref. 09/H0605/60) approved the study. All participants gave written consent. Fifty subjects (18 males; mean age $=28.93 \pm 7.7$ years) were recruited from the general population, based on neuroticism scores on the Eysenck Personality Questionnaire (EPQ) (Eysenck and Eysenck, $1975)$ either low $(\leqslant 6)$ or high $(\geqslant 16)$, approximately corresponding to the mean neuroticism scores of the same aged general population (Eysenck et al, 1985) + 1 SD, which conveys an 50-60\% increase in lifetime risk for developing depression (Kendler et al, 1993). Participants were screened for axis I disorders using the Structured Clinical Interview for DSM-IV (First et al, 1996) and completed the State-Trait Anxiety Inventory (Spielberger et al, 1983), Beck Depression Inventory (Beck et al, 1961), Dysfunctional Attitude Scale (Weissman and Beck, 1978), and visual analogue scales (VAS) rating happiness, sadness, hostility, alertness, anxiety, and calmness. Three subjects were excluded owing to history of depression (before data collection, all from the high neuroticism group) and three owing to bad quality of data recording (two from the high and one from the low neuroticism group). The final sample comprised 44 subjects (17 males, mean age $=29.6 \pm 7.9$ years), of which 24 had low neuroticism scores (Low Ns) (10 males, mean $N=2.62 \pm$ 1.4) and 20 had high neuroticism scores (High Ns) (7 males, mean $N=18.45 \pm 2.2$ ). Gender imbalance in the High Ns group reflects the personality trait distribution in the general population (Lynn and Martin, 1997).

Gender discrimination task. A task previously adopted in fMRI experiments with a similar sample was used (Chan et al, 2009; Di Simplicio et al, 2013). Participants were presented with 8 faces on a computer displaying prototypical expressions of fear and happiness, and a neutral face (29) morphed at 30\% (low), 60\% (medium), and 100\% (high intensity) of the expression along the neutral-prototypic continuum. A total 168 stimuli were presented in a random order for $500 \mathrm{~ms}$ each, with intertrial interval varying according to a Poisson distribution (mean $=5000 \mathrm{~ms}$ ). Participants indicated the gender of each face by a key press. Behavioral responses (accuracy and RT) were recorded using Presentation (Neurobehavioral Systems, Albany, CA).

Eye-movement recording. During the task, eye movements were recorded using an infrared eye-tracking system (ISCAN, Woburn, MA) at a rate of $60 \mathrm{~Hz}$. A chin rest ensured a stable position of the head at $100-\mathrm{cm}$ screen distance. Eye position was calibrated using a five-point calibration procedure.

Data were analyzed using the i-LAB software (Gitelman, 2002) under the Matlab environment (Mathworks, Inc.).

For each trial, blinks were filtered using the pupil-size parameter, and missing points were replaced using a linear interpolation between preblink and postblink values. To derive eye-movement measures, three rectangular regions of interest (ROIs) were drawn covering: (1) the eye region, (2) the mouth and lower nose region, and (3) the whole face. 
Parameters were computed as the proportion of ocular movements made over eyes/mouth ROI within the total ocular movements made over the whole face.

Four dependent measures were extracted for analysis: number of fixations, scanpath length, scanning time, and gaze maintenance. Fixations represent the frequency of stationary gaze points during scanning and were defined as a set of eye positions within a defined area $(0.30 \times 0.30$ degree area) for a selected amount of time $(100 \mathrm{~ms})$ and calculated using a velocity/distance algorithm (Gitelman, 2002). Scanpath length refers to the summed distances traveled by the eye during scanning, measured in degrees of visual angle (expressed in pixels). Scanning time refers to the time spent by the eye traveling over the stimuli, calculated in ms. The gaze maintenance calculation indicated whether gaze was or not maintained in the selected ROI for the entire trial duration $(500 \mathrm{~ms})$.

For each outcome measure, a cutoff of minimum $25 \%$ valid trials was adopted in order for data from any individual to be included.

Statistical analysis. Box plots were used to identify outliers. Values $>3$ box-lengths from the 75 th percentile (box-length $=$ interquartile range) were excluded.

Behavioral data (gender discrimination mean accuracy and mean RT) were analyzed using repeated-measures ANOVAs, with group (High vs Low Ns) as the betweensubject variable and emotion (fearful, happy) and intensity of facial expression (high, medium, low) as the withinsubjects variables. The eye-movement parameters were analyzed using repeated-measures ANOVAs, first over the whole face ROI with group (High vs Low Ns) as the between-subject variable and emotion (fearful, happy) and intensity of facial expression (high, medium, low) as the within-subjects variables. Ocular exploration over different face areas was analyzed, including the additional withinsubjects variable of within-face ROIs (eyes, mouth) to the repeated-measures ANOVAs. The interpretation of significant interaction effects was aided by the use of post hoc analysis (Bonferroni) to compare between levels of the different factors and by use of simple main-effect analyses. Independent samples $t$-tests were performed to examine group differences in affect and personality ratings. Correlation analyses were run to check for relationships between eye-movement parameters and behavioral responses and affect ratings in the High Ns group. In order to simplify correlation analysis, eye-movement data were collapsed across different facial expression intensities.

\section{STUDY 2 Ocular Exploration of Emotional Facial Expression: 7 Days Citalopram vs Placebo}

Volunteers and design. Recruitment and screening measures were the same as in Study 1, but only individuals with High Ns were selected ( $\geqslant 16$ on neuroticism scale of the EPQ) (Eysenck and Eysenck, 1975). Forty-seven participants were recruited from the general population; five were excluded owing to history of depression (before data collection and randomized group allocation), and six owing to bad quality of data recording (after data collection; four from the citalopram, two from the placebo group). Three more participants ( 2 from the citalopram, 1 from the placebo group) were excluded as outliers (see definition above), leaving a total sample of 33 (20 males, mean age $=23.6 \pm 6.1$ years), of which 16 received citalopram (10 males, mean $N=18.44 \pm 2.4$ ) and 17 placebo (10 males, mean $N=19.76 \pm 1.8$ ). Participants were randomly allocated to receive identical capsules of citalopram $20 \mathrm{mg}$ or placebo, instructed to take them every day after breakfast, and tested on the seventh day of administration, avoiding caffeinated drinks from $2 \mathrm{~h}$ before testing.

Gender discrimination task and eye-movement recording. The experimental methods were the same as in Study 1.

Facial expression recognition task (FERT). In this study, participants performed an additional task (FERT) previously shown to be sensitive to antidepressant manipulation (Harmer, 2008), where subjects are asked to label emotional facial expressions presented on a screen for $500 \mathrm{~ms}$ at intensities varying between 10 and 100\%. Correct responses and misclassifications are recorded, and accuracy is measured calculating the average expression intensity threshold needed to correctly identify each emotion (Chan et al, 2007). Eye movements were not recorded during the FERT.

Statistical analysis. The analysis methods were the same as in Study 1. In the repeated-measures ANOVAs, treatment group (drug vs placebo) was entered as the between-subjects variable. A correlation analysis was also run between eyemovement parameters and facial expression intensity threshold values for correct emotion recognition on the FERT, to explore whether emotion recognition accuracy was related to patterns of ocular face exploration.

\section{RESULTS}

For both studies, results from personality and affect ratings, and behavioral performance on the gender discrimination task, are reported in the Supplementary Material (Supplementary Tables S1-S4). Eye-movement data are only reported for the ocular measures with sufficient valid trials and for which significant between-group differences were found. All $p$-values reported are Bonferroni corrected for multiple comparisons.

\section{STUDY 1 Ocular Exploration of Emotional Facial Expression: High $v s$ Low Neuroticism}

\section{Gender discrimination task: eye movements}

Whole face area. Recording of eye movements did not show any significant between-group differences in ocular exploration of different emotional expressions (all $p$ 's $>0.1$ ).

Eyes vs mouth-gaze maintenance. In the emotion $\times$ intensity $\times$ ROI $\times$ group ANOVA analyzing gaze maintenance over different areas of the facial expressions, a significant ROI (eyes $v s$ mouth) $\times$ group (High $v s$ Low Ns) interaction was present $(\mathrm{F}(1,42)=10.328, p=0.003$; Table 1$)$. This showed that High Ns spent less time gazing at the eyes region and more at the mouth region of faces compared with Low Ns (eyes ROI: $\mathrm{F}(1,42)=4.949, p=0.032$; mouth ROI: $\mathrm{F}(1,42)=10.190, p=0.003$; Figure 1$)$. 
Table I Eye Movements Over Facial Expressions During a Gender Discrimination Task in Subjects with Low (Low Ns, $N=24$ ) and High Neuroticism (High Ns, N=20) Scores: Average Presence of Eye Gaze Maintenance over a Face Area for the Whole Trial Duration

\begin{tabular}{lcc}
\hline $\begin{array}{l}\text { Eye gaze maintenance presence } \\
\text { over whole trial duration }\end{array}$ & $\begin{array}{c}\text { Low Ns } \\
\text { (Mean } \pm \text { SD) }\end{array}$ & $\begin{array}{c}\text { High Ns } \\
\text { (Mean } \pm \text { SD) }\end{array}$ \\
\hline $\begin{array}{l}\text { Eye area } \\
\text { High fearful }\end{array}$ & $0.29 \pm 0.35$ & $0.10 \pm 0.22$ \\
Medium fearful & $0.30 \pm 0.34$ & $0.10 \pm 0.22$ \\
Low fearful & $0.27 \pm 0.30$ & $0.10 \pm 0.24$ \\
High happy & $0.28 \pm 0.33$ & $0.10 \pm 0.22$ \\
Medium happy & $0.27 \pm 0.32$ & $0.11 \pm 0.25$ \\
Low happy & $0.30 \pm 0.32$ & $0.09 \pm 0.23$ \\
Neutral & $0.30 \pm 0.32$ & $0.11 \pm 0.24$ \\
& & \\
Mouth area & & \\
High fearful & $0.15 \pm 0.27$ & $0.48 \pm 0.42$ \\
Medium fearful & $0.13 \pm 0.26$ & $0.44 \pm 0.40$ \\
Low fearful & $0.13 \pm 0.26$ & $0.47 \pm 0.40$ \\
High happy & $0.17 \pm 0.29$ & $0.48 \pm 0.44$ \\
Medium happy & $0.16 \pm 0.27$ & $0.48 \pm 0.41$ \\
Low happy & $0.12 \pm 0.26$ & $0.49 \pm 0.42$ \\
Neutral & $0.12 \pm 0.25$ & $0.44 \pm 0.42$ \\
\hline
\end{tabular}

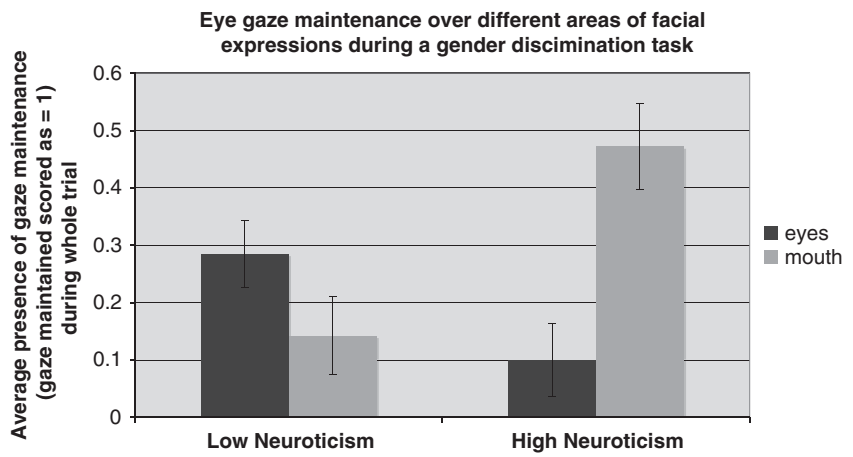

Figure I Subjects with High Neuroticism scores maintain less frequently the gaze over the eye region and more over the mouth region for the whole duration of a trial compared with subjects with Low Neuroticism scores.

Correlations with behavioral performance and affect ratings. Correlation analysis between gaze parameters and affect ratings before testing revealed a significant negative correlation between self-ratings of hostility on VAS and gaze maintenance over the eye region of both happy $(R=-0.598, p=0.007)$ and fearful faces $(R=-0.640$, $p=0.002)$ in the High Ns group.

\section{STUDY 2 Ocular Exploration of Emotional Facial Expression: 7 Days Citalopram vs Placebo}

FERT. To compare accuracy at labeling positive $v s$ negative emotions, thresholds for correct recognition were aggregated
Table 2 Eye Movements Over Facial Expressions During a Gender Discrimination Task, After 7 Days Treatment with Citalopram or Placebo in Subjects with High Neuroticism Scores

\begin{tabular}{lll}
\hline & $\begin{array}{c}\text { Citalopram } \\
\text { (Mean } \pm \text { SD) }\end{array}$ & $\begin{array}{c}\text { Placebo } \\
\text { (Mean } \pm \text { SD) }\end{array}$ \\
\hline $\begin{array}{l}\text { Scanpath lenghth }(p x l) \\
\text { High fearful }\end{array}$ & \\
Medium fearful & $46.54 \pm 32.75$ & $51.31 \pm 21.37$ \\
Low fearful & $44.28 \pm 33.48$ & $65.52 \pm 32.27$ \\
High happy & $43.72 \pm 28.54$ & $45.61 \pm 18.15$ \\
Medium happy & $49.43 \pm 28.86$ & $50.84 \pm 16.55$ \\
Low happy & $46.37 \pm 30.16$ & $51.55 \pm 28.1$ \\
Neutral & $41.37 \pm 30.80$ & $51.52 \pm 27.53$ \\
& $41.71 \pm 22.22$ & $46.14 \pm 17.99$ \\
Scanning time (ms) & & \\
High fearful & & $210.39 \pm 36.82$ \\
Medium fearful & $239.53 \pm 5$ & $212.76 \pm 32.97$ \\
Low fearful & $238.95 \pm 5.93$ & $217.51 \pm 31.21$ \\
High happy & $240.43 \pm 4.52$ & $209.11 \pm 32.69$ \\
Medium happy & $235.59 \pm 11.6$ & $213.89 \pm 35.32$ \\
Low happy & $238.41 \pm 6.78$ & $206.88 \pm 45.03$ \\
Neutral & $235.83 \pm 13.75$ & $208.62 \pm 38.96$
\end{tabular}

Gaze maintenance presence over whole trial duration

\begin{tabular}{lrr} 
High fearful & $0.98 \pm 0.04$ & \multicolumn{1}{c}{$0.9 \pm 0.1$} \\
Medium fearful & $0.99 \pm 0.02$ & $0.88 \pm 0.13$ \\
Low fearful & $1 \pm 0.01$ & $0.92 \pm 0.07$ \\
High happy & $0.95 \pm 0.07$ & $0.89 \pm 0.11$ \\
Medium happy & $0.98 \pm 0.04$ & $0.9 \pm 0.08$ \\
Low happy & $0.98 \pm 0.05$ & $0.86 \pm 0.17$ \\
Neutral & $0.98 \pm 0.04$ & $0.88 \pm 0.11$
\end{tabular}

Eye movement parameters indicate the average area explored over each face type (scanpath length), the average time spent scanning each face type (scanning time), and whether eye gaze was maintained fixed over each face type for the whole duration of a trial (gaze maintenance).

for negative (sad, angry, and fearful) and positive (happy, surprised) facial emotional expressions. There was a significant valence $\times$ treatment group interaction $(F(1,36)=5.60$, $p=0.023$ ), with subjects on citalopram requiring a lower intensity of emotional expression to correctly recognize positive $v s$ negative emotions compared with subjects on placebo. Detailed task results are reported in Supplementary Materials (Supplementary Table S5).

Gender discrimination task: eye movements. Mean results for each group are described in Table 2.

Whole face area-gaze maintenance. In the emotion $\times$ intensity $\times$ group ANOVA analyzing gaze maintenance, a significant between-group difference was found irrespective of facial expression and intensity, driven by subjects on citalopram maintaining their gaze over the faces for the whole 


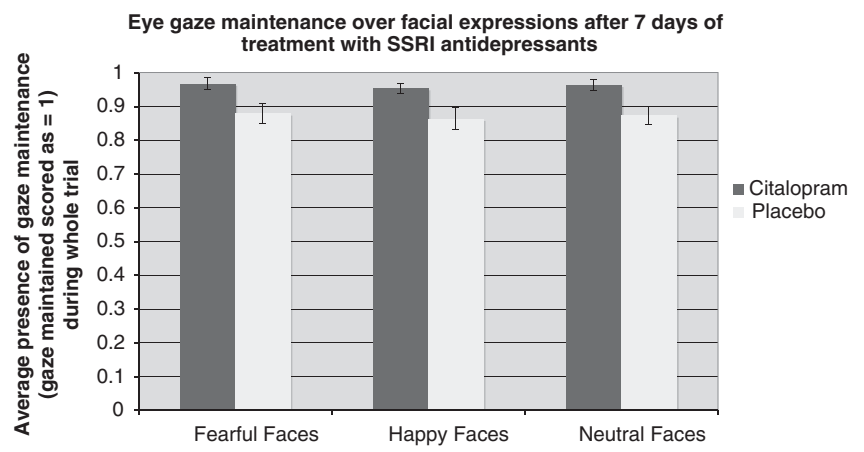

Figure 2 Citalopram-treated subjects with High Neuroticism maintain the gaze more over all faces for the whole trial duration during a gender discrimination task, compared with those on placebo.

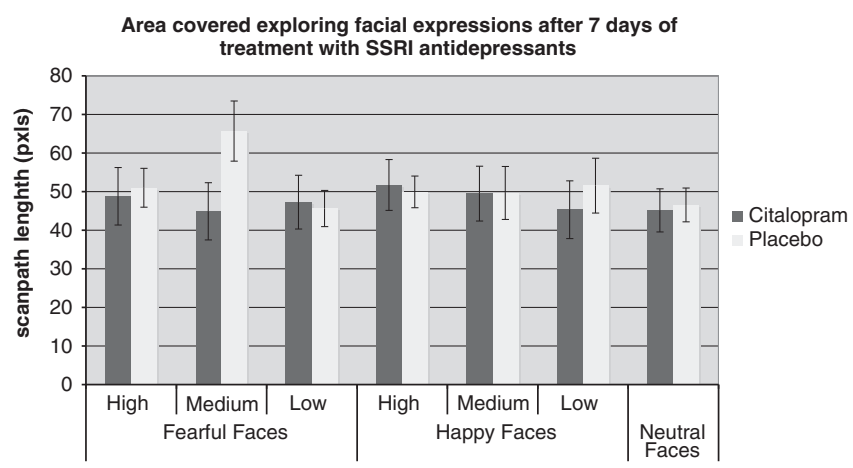

Figure 3 Citalopram-treated subjects with High Neuroticism explore smaller extensions of fearful faces with medium emotional intensity compared with those on placebo.

trial duration more than subjects on placebo (main effect of group: $\mathrm{F}(1,31)=11.664, p=0.002$; Figure 2 ).

Whole face area-scanpath length. In the emotion $\times$ intensity $\times$ group ANOVA analyzing the scanpath length, all subjects showed larger degree of exploration of faces with medium emotional intensity $(\mathrm{F}(2,62)=6.024, p=0.004)$. A significant two-way interaction was found between group and intensity of facial expression $(\mathrm{F}(2,62)=3.651$, $p=0.032$ ), such that within the placebo but not within the citalopram-treated group participants showed larger degree of spatial exploration of medium compared with low and high intensity faces (placebo: $\mathrm{F}(2,62)=6.372, p=0.005$; drug: $\mathrm{F}(2,62)=2.038, p=0.149)$. A significant three-way interaction was also present between group, emotion, and intensity of facial expression $(\mathrm{F}(2,62)=3.692, p=0.031$, (Cohen's $d=0.64)$ ), indicating that individuals on citalopram tended to spatially explore the fearful faces of medium intensity less compared with individuals on placebo $(\mathrm{F}(1,31)=3.255, p=0.083)$ (Figure 3$)$.

Whole face area-scanning time. In the emotion $\times$ intensity $\times$ group ANOVA analyzing the amount of time spent scanning faces, a significant between-group difference was found irrespective of facial expression and intensity, driven by subjects on citalopram scanning all faces longer compared with subjects on placebo $(\mathrm{F}(1,31)=9.867$, $p=0.004$ ) (Supplementary Figure S1).

Eyes vs mouth-gaze maintenance. In the emotion $\times$ intensity $\times$ ROI $\times$ group ANOVA analyzing the proportion of gaze maintenance over different areas of the facial expressions, a significant main effect of group was found driven by subjects on citalopram maintaining their gaze for the whole trial duration over both eyes and mouth areas of all faces more than subjects on placebo $(\mathrm{F}(1,31)=6.378$ $p=0.017)$.

Correlations with behavioral performance and affect ratings. A significant positive correlation was observed between gender discrimination accuracy for fearful faces and scanning time $(R=0.374, p=0.027)$. Instead, a negative correlation was found between gender discrimination accuracy for fearful faces and scanpath length $(R=-0.366, p=$ 0.031 ). No correlations were found with affect ratings.

Correlations with emotion recognition. A significant negative correlation was found between the intensity threshold needed to correctly recognize happy faces and gaze maintenance over the whole area of happy facial expression $(R=-0.358, p=0.044)$ and between the number of misclassifications of happy faces and both gaze maintenance and scanning time over the whole area of happy facial expression $(R=-0.518, p=0.002 ; R=-0.473, p=0.006)$. Given the significant between-group difference in facial expression recognition, driven by the citalopram-treated group performing better on positive faces, an exploratory regression analysis was also run to test whether emotion recognition was predicted by the pattern of ocular exploration of the faces. A significant predictive model was found with recognition threshold of positive faces as the dependent variable and group, ocular scanning time over happy faces, and the interaction between group and scanning time as independent variables $(R=0.503, \mathrm{dF}=(3,31), \mathrm{F}=3.157$, $p=0.040)$. Scanning time over happy faces and the interaction between group and scanning time over happy faces were significant predictors of positive emotion recognition performance $(\beta=-1.366, p=0.018$ and $\beta=1.181, p=0.021$; Supplementary Figure S2).

\section{DISCUSSION}

This study investigated whether neuroticism affects ocular exploration of emotional faces and whether this can be modulated by antidepressant drug treatment. Using a gender-discrimination task of fearful and happy faces with different emotional intensity, we found that: (a) subjects with high neuroticism present a different pattern of ocular exploration of faces compared with subjects with low neuroticism, characterized by reduced gaze maintenance over the eyes region of the face, irrespective of emotional expression; (b) short-term repeated SSRI administration increased eye-gaze maintenance and scanning time over all facial expressions, irrespective of emotion type and intensity, compared with placebo in a high-risk sample with high neuroticism. The same treatment also reduced the 
spatial extension explored by eye movements over faces of medium emotional intensity, and this was significant for fearful faces. Moreover, (c) eye-movement pattern predicted performance on facial expression recognition on a separate task. These results occurred in the absence of any subjective modifications in mood or anxiety (Supplementary Material), consistent with the model by which antidepressants' action on the processing of emotional information precedes changes in affective state (Pringle et al, 2011).

\section{Effects of High Neuroticism on Ocular Exploration of Face Stimuli}

Our study indicates that high neuroticism is characterized by an attentional bias away from salient face regions, which is not affected by emotional valence of the facial expression and is indexed by an abnormal pattern of ocular face exploration. High Ns avoided holding their gaze over the eye region for the whole trial duration, and general face avoidance was significantly correlated with self-rated hostility. This suggests that High Ns may avoid engaging with socially salient stimuli such as faces, reflecting a selfperceived negative disposition towards interpersonal contact. Previous studies have reported a similar pattern in socially phobic patients (Horley et al, 2003). Although we did not collect measures of social anxiety, stress during social interactions is a well-recognized feature of the high neurotic phenotype, contributing to the higher risk for social anxiety (Clark et al, 1994; Watson et al, 2008). Interestingly, this avoidant ocular exploration pattern is consistent with reduced neural response to faces (irrespective of emotional expression) in the amygdala in the same population (Di Simplicio et al, 2013).

Differences in the task demands could explain the discrepancy between our findings and that by Perlman et al (2009), who reported that increased scanning over the eye region, in particular of fearful faces, is associated with high neuroticism during an overt emotion discrimination task. It is possible that when explicitly cued to respond to the emotional expression of the face, highly neurotic subjects can orient their attention to the eyes, which they otherwise avoid when less relevant to their contingent objective. If this were true, the increased eye region exploration over fearful eyes found by Perlman et al (2009) could represent a task-driven strategy, consistent with a recent meta-analysis showing that heterogeneity of results from attention orienting and maintenance data is often context dependent (Armstrong and Olatunji, 2012). Another explanation could lie in our sample focused on High N individuals, whereas Perlman et al (2009) using a continuous measure: 'extreme' neuroticism, is more likely to present prominent socially anxious features, which could explain an avoidant pattern of eye region exploration (Gamble and Rapee, 2010).

Together, these data suggest that high neuroticism is associated with inadequate capacity to maintain attention over key areas of face stimuli, which could in turn underpin interpersonal difficulties often presented by this group. This may contribute to increased risk of developing anxiety consistent with clinical models (Clark and Wells, 1995) and to lower quality of life, influenced by hypersensitivity to interpersonal stressors and lack of social support (Kendler and Gardner, 2014; Lahey, 2009). Of note, neuroticism is a heterogeneous and multifaceted construct. Our task of covert attention towards emotional facial expression with a short stimulus presentation allows a global assessment of emotion-processing biases, more in particular to those related to general anxiety (hypervigilance-avoidance of negative stimuli) and social anxiety (avoidance of the eye region of faces). Future studies could employ larger samples and identify population subgroups with high neuroticism characterized by more prominent dysphoric, generally anxious, and socially anxious traits. This could be combined with tasks showing concurrent negative and positive facial expressions and longer stimulus presentation in order to investigate more extensively the presence of attentional biases associated with anxiety and depression, respectively.

\section{Effect of SSRIs on Ocular Exploration of Face Stimuli}

We also found that SSRIs can modulate attentional biases towards faces in a high-risk sample early in treatment, by directly increasing gaze maintenance over the internal features of faces. This suggests that an initial SSRIs' effect in anxious individuals could be to partly correct their dysfunctional pattern of ocular exploration of faces.

Initial attention allocation to salient stimuli is associated with amygdala activation (Vuilleumier, 2005). The present finding is therefore consistent with neuroimaging data showing that short-term repeated SSRI administration to High Ns increased the amygdala signal to facial expression irrespective of emotion type (Di Simplicio et al, 2013). The causal mechanism operating between amygdala activation and eye gaze movements remains unclear. The first neural response could precede attention deployment, guiding the eyes to explore the detected stimuli (Kennedy and Adolphs, 2011), but time spent gazing over the stimuli could equally increase the limbic neural signal (Pessoa and Adolphs, 2010). SSRI-driven modifications could occur at one or both stages of this process.

Notably, in the citalopram-treated group prolonged attention to faces (as measured by the ability to hold the gaze on the face for the entire trial and by the time spent scanning faces) was predictive of better performance in the gender discrimination task and to a more accurate discrimination of positive expressions in a separate facial expressions recognition task. High Ns find happiness recognition particularly difficult compared with non-vulnerable samples (Chan et al, 2007), and the current data suggest that recognition of positive emotional facial expressions improves early with SSRI treatment in this group. Although our data cannot make any causal inference, they suggest that modulation of basic eye-movement and face-exploration parameters by SSRIs are associated with correction of this negative bias in emotion recognition. Further investigations should confirm whether the capacity of different antidepressant agents to shift biases in facial expression recognition in unselected populations (Pringle et al, 2011) is similarly associated with effects on ocular exploration.

By contrast, short-term repeated SSRI administration did not modify the preference for exploring the mouth rather than the eye region seen in High Ns in Study 1. This suggests that antidepressants may produce a non-specific effect on gaze maintenance over faces. In a previous 
research, 2 weeks of citalopram administration in healthy volunteers increased eye contact compared with placebo in an interaction task with a stranger (Tse and Bond, 2002). Future studies with samples also characterized on social anxiety dimensions, more naturalistic experimental settings, and longer treatment duration could clarify whether the SSRI-mediated increase in face exploration after 1 week would also facilitate with time the reversal of the eye region avoidance when this is present.

Interestingly, citalopram-treated individuals also tended to spacially explore the face area less compared with placebo-treated ones, but this effect appeared prominent for fearful faces of medium intensity. Anxious individuals have been shown to spatially explore larger areas of faces while holding the eye gaze for shorter time duration over the face (Horley et al, 2003). This could reflect a 'scanning style' of faces that avoids to properly stop over those cues holding potential danger and can impair accurate processing (Armstrong and Olatunji, 2012). This result should be treated with caution given the relatively small sample, with a medium effect size of the comparison. Accordingly, in our sample the spatial extension of eye movements was negatively correlated with gender discrimination accuracy. Hence, the combination of SSRI-induced changes in ocular exploration over fearful expressions and over all faces could lead to a more functional attention deployment toward socially salient stimuli, contributing to the long-term anxiolytic effect of antidepressants. If this were true, antidepressant action would appear to occur via an 'exposure' mechanism similar to cognitive-behavioral treatments, as we have previously suggested (Di Simplicio et al, 2013).

\section{Summary}

In summary, our results demonstrate that short-term antidepressant administration can modify the pattern of attention for facial stimuli by changing ocular movements exploring faces in subjects at risk for depression and anxiety, before any changes in affect. Our data illustrate how antidepressant medication could reduce the difficulties in extracting information from facial stimuli by increasing evaluative capacity. This can have different clinical impact depending on preexisting attentional biases. It might temporarily increase anxiety in subjects hypervigilant for threat, but it can also increase positive affect in subjects generally avoidant of social contacts and thus deprived of positive interpersonal feedback. This is consistent with the variable responses to SSRI treatment in clinical practice, including the so-called early 'anxiogenic effects' (Sinclair et al, 2009; Yeragani et al, 1992). Further studies will need to verify whether our findings can be extended to clinical populations and to investigate how modification of ocular exploration of socially salient stimuli influences remission of anxious and depressive symptoms over time.

\section{FUNDING AND DISCLOSURE}

The study was supported by a Medical Research Council grant to Professor Catherine Harmer. Dr Martina Di Simplicio was supported by the doctoral research program in Applied Neurosciences of the University of Siena, Italy. Sonia Doallo was supported by a postdoctoral contract from the Isidro Parga Pondal program (Xunta de Galicia, Spain). Professor Catherine Harmer is a company director of Oxford Psychologists and has received consultancy or speaker fees from P1vital, Servier, Lundbeck, and Eli-Lilly. Professor Catherine Harmer is on the advisory panel and holds shares in P1vital. The other authors declare no conflict of interest.

\section{ACKNOWLEDGEMENTS}

We thank all participants in the study.

\section{REFERENCES}

Armstrong T, Olatunji BO (2012). Eye tracking of attention in the affective disorders: a meta-analytic review and synthesis. Clin Psychol Rev 32: 704-723.

Beck AT, Ward CH, Mendelson M, Mock J, Erbaugh J (1961). An inventory for measuring depression. Arch Gen Psychiatry 4: 561-571.

Browning M, Holmes EA, Harmer CJ (2010). The modification of attentional bias to emotional information: a review of the techniques, mechanisms, and relevance to emotional disorders. Cogn Affect Behav Neurosci 10: 8-20.

Butler G, Fennell M, Hackman A (2008). Avoidance of Affect. The Guilford Press: New York, NY, USA.

Canli T (2008). Toward a neurogenetic theory of neuroticism. Ann NY Acad Sci 1129: 153-174.

Caseras X, Garner M, Bradley BP, Mogg K (2007). Biases in visual orienting to negative and positive scenes in dysphoria: An eye movement study. J Abnorm Psychol 116: 491-497.

Chan SW, Goodwin GM, Harmer CJ (2007). Highly neurotic neverdepressed students have negative biases in information processing. Psychol Med 37: 1281-1291.

Chan SW, Norbury R, Goodwin GM, Harmer CJ (2009). Risk for depression and neural responses to fearful facial expressions of emotion. Br J Psychiatry 194: 139-145.

Clark DM, Wells A (1995). A cognitive Model of Social Phobia. The Guildford Press: New York, NY, USA.

Clark LA, Watson D (1991). Tripartite model of anxiety and depression: psychometric evidence and taxonomic implications. J Abnorm Psychol 100: 316-336.

Clark LA, Watson D, Mineka S (1994). Temperament, personality, and the mood and anxiety disorders. J Abnorm Psychol 103: $103-116$.

Derakshan N, Salt M, Koster EH (2009). Attentional control in dysphoria: an investigation using the antisaccade task. Biol Psychol 80: 251-255.

Di Simplicio M, Norbury R, Harmer CJ (2012). Short-term antidepressant administration reduces negative self-referential processing in the medial prefrontal cortex in subjects at risk for depression. Mol Psychiatry 17: 503-510.

Di Simplicio M, Norbury R, Reinecke A, Harmer CJ (2013). Paradoxical effects of short-term antidepressant treatment in fMRI emotional processing models in volunteers with high neuroticism. Psychol Med 44: 1-12.

Eysenck SBG, Eysenck HJ (1975). Manual of the EPQ (Eysenck Personality Questionnaire). University of London Press: London, UK.

Eysenck SBG, Eysenck HJ, Barrett P (1985). A revised version of the psychoticism scale. Pers Individ Dif 6: 21-29.

First MD, Spitzer RL, Gibbon M, Williams JB (1996). Structured Clinical Interview for DSM-IV Axis I Disorders (SCID). American Psychiatric Press, Inc: Washington, DC, USA. 
Gamble AL, Rapee RM (2010). The time-course of attention to emotional faces in social phobia. J Behav Ther Exp Psychiatry 41: 39-44.

Garner M, Mogg K, Bradley BP (2006). Orienting and maintenance of gaze to facial expressions in social anxiety. J Abnorm Psychol 115: $760-770$.

Gitelman DR (2002). ILAB: a program for postexperimental eye movement analysis. Behav Res Methods Instrum Comput 34: 605-612.

Godlewska BR, Norbury R, Selvaraj S, Cowen PJ, Harmer CJ (2012). Short-term SSRI treatment normalises amygdala hyperactivity in depressed patients. Psychol Med 42: 2609-2617.

Gotlib IH, Krasnoperova E, Yue DN, Joormann J (2004). Attentional biases for negative interpersonal stimuli in clinical depression. J Abnorm Psychol 113: 121-135.

Harmer CJ (2008). Serotonin and emotional processing: does it help explain antidepressant drug action? Neuropharmacology 55: 1023-1028.

Harmer CJ, Mackay CE, Reid CB, Cowen PJ, Goodwin GM (2006). Antidepressant drug treatment modifies the neural processing of nonconscious threat cues. Biol Psychiatry 59: 816-820.

Horley K, Williams LM, Gonsalvez C, Gordon E (2003). Social phobics do not see eye to eye: a visual scanpath study of emotional expression processing. J Anxiety Disord 17: 33-44.

Itier RJ, Batty M (2009). Neural bases of eye and gaze processing: the core of social cognition. Neurosci Biobehav Rev 33: 843-863.

Kendler KS, Gardner CO (2014). Sex differences in the pathways to major depression: a study of opposite-sex twin pairsAm J Psychiatry.

Kendler KS, Neale MC, Kessler RC, Heath AC, Eaves LJ (1993). A longitudinal twin study of personality and major depression in women. Arch Gen Psychiatry 50: 853-862.

Kennedy DP, Adolphs R (2011). Reprint of: Impaired fixation to eyes following amygdala damage arises from abnormal bottomup attention. Neuropsychologia 49: 589-595.

Kent JM, Coplan JD, Gorman JM (1998). Clinical utility of the selective serotonin reuptake inhibitors in the spectrum of anxiety. Biol Psychiatry 44: 812-824.

Lahey BB (2009). Public health significance of neuroticism. Am Psychol 64: 241-256.

Leyman L, De Raedt R, Vaeyens R, Philippaerts RM (2011). Attention for emotional facial expressions in dysphoria: an eyemovement registration study. Cogn Emot 25: 111-120.

Lynn R, Martin T (1997). Gender differences in extraversion, neuroticism, and psychoticism in 37 nations. J Soc Psychol 137: 369-373.

Murphy SE, Yiend J, Lester KJ, Cowen PJ, Harmer CJ (2009). Shortterm serotonergic but not noradrenergic antidepressant administration reduces attentional vigilance to threat in healthy volunteers. Int J Neuropsychopharmacol 12: 169-179.
Nolen-Hoeksema S (2000). The role of rumination in depressive disorders and mixed anxiety/depressive symptoms. J Abnorm Psychol 109: 504-511.

Ormel J, Rosmalen J, Farmer A (2004). Neuroticism: a noninformative marker of vulnerability to psychopathology. Soc Psychiatry Psychiatr Epidemiol 39: 906-912.

Ouimet AJ, Gawronski B, Dozois DJ (2009). Cognitive vulnerability to anxiety: a review and an integrative model. Clin Psychol Rev 29: 459-470.

Perlman SB, Morris JP, Vander Wyk BC, Green SR, Doyle JL, Pelphrey KA (2009). Individual differences in personality predict how people look at faces. PLoS One 4: e5952.

Pessoa L, Adolphs R (2010). Emotion processing and the amygdala: from a 'low road' to 'many roads' of evaluating biological significance. Nat Rev Neurosci 11: 773-783.

Pringle A, Browning M, Cowen PJ, Harmer CJ (2011). A cognitive neuropsychological model of antidepressant drug action. Prog Neuropsychopharmacol Biol Psychiatry 35: 1586-1592.

Sinclair LI, Christmas DM, Hood SD, Potokar JP, Robertson A, Isaac A et al (2009). Antidepressant-induced jitteriness/anxiety syndrome: systematic review. Br J Psychiatry 194: 483-490.

Spielberger CD, Gorsuch RL, Lushene RD (1983). Manual for the State-Trait Anxiety Inventory (STAI). Consulting Psychologists Press: Palo Alto, CA, USA.

Tse WS, Bond AJ (2002). Serotonergic intervention affects both social dominance and affiliative behaviour. Psychopharmacology 161: 324-330.

Vuilleumier P (2005). How brains beware: neural mechanisms of emotional attention. Trends Cogn Sci 9: 585-594.

Watson D, Clark LA, Chmielewski M (2008). Structures of personality and their relevance to psychopathology: II. Further articulation of a comprehensive unified trait structure. J Pers 76: 1545-1586.

Weissman AN, Beck AT (1978). Development and validation of the dysfunctional attitude scale. Annual Meeting of the Association for the Advanced Behavior Therapy. Chicago, IL, USA.

Whalen PJ, Kagan J, Cook RG, Davis FC, Kim H, Polis S et al (2004). Human amygdala responsivity to masked fearful eye whites. Science 306: 2061.

Yeragani VK, Pohl R, Balon R, Ramesh C, Weinberg P (1992). Imipramine-induced jitteriness and decreased serum iron levels. Neuropsychobiology 25: 8-10.

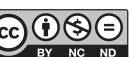

This work is licensed under a Creative Commons Attribution-NonCommercial-NoDerivs $3.0 \mathrm{Un}$ ported License. To view a copy of this license, visit http:// creativecommons.org/licenses/by-nc-nd/3.0/

Supplementary Information accompanies the paper on the Neuropsychopharmacology website (http://www.nature.com/npp) 